\title{
A risk factor analysis on disease severity in 47 premature infants with bronchopulmonary dysplasia
}

\author{
Yan $\mathrm{Li}^{1,2}$, Yazhou Cui ${ }^{1}$, Chao Wang ${ }^{1,2}$, Xiao Liu ${ }^{1,2}$, Jinxiang Han ${ }^{1, *}$ \\ ${ }^{1}$ Key Laboratory for Biotech Drugs of the Ministry of Health, Key Laboratory for Rare Disease of Shandong Province, Shandong \\ Medicinal Biotechnology Center, Shandong Academy of Medical Sciences, Ji'nan, Shandong, China; \\ ${ }^{2}$ School of Medicine and Life Sciences, University of Jinan-Shandong Academy of Medical Science, Ji'nan, Shandong, China.
}

\begin{abstract}
Summary Bronchopulmonary Dysplasia (BPD) is a rare chronic lung disease and one of the most difficult complications to treat in premature infants. With the progress at the medical treatment level, an increasing number of BPD premature infants are born, meanwhile, they would be at an increasing risk for numerous complications and rehospitalization because BPD affects many vital organ systems. The pathogenesis of BPD is clearly multifactorial. As the prognosis is closely connected with the severity of BPD, early diagnosis and treatment are of great help to control the development of BPD. This article focuses on risk factors that could influence the severity of BPD in order to provide a reliable basis for early diagnosis, treatment, and better patient assessment.
\end{abstract}

Keywords: Bronchopulmonary dysplasia, preterm infants, risk factors

\section{Introduction}

Bronchopulmonary dysplasia (BPD) is a rare chronic lung disease in premature infants resulting from oxygen and mechanical ventilation that was first described by Northway et al. in 1967 (1). BPD has become one of the thorniest issues in the neonatal intensive care unit (NICU) and the main cause of chronic respiratory diseases of infants due to auxiliary oxygen for a long time, a high mortality rate, survivors with high reactive airway disease, feeding difficulties and growth retardation. Children with BPD need long-term dependence on oxygen which, easily causes repeated infection of the lung and even leads to physical and intellectual developmental disorders. BPD seriously affects the survival rate and living quality of premature infants and, it brings a heavy burden to the family and society. The reported incidence of BPD was quite different in various studies. Possible reasons were

Released online in J-STAGE as advance publication March 31, 2015.

*Address correspondence to:

Dr. Jinxiang Han, Key Laboratory for Rare Disease Research of Shandong Province, Key Laboratory for Biotech Drugs of the Ministry of Health, Shandong Medical Biotechnological Center, Shandong Academy of Medical Sciences, Ji'nan, Shandong 250062, China.

E-mail: samshjx@sina.com differences in clinical definitions (2), demographics of patient populations and management strategies used across studies (3). There are about 3,000 7,000 newborns suffering from BPD in America every year (4), however, the morbidity is not clear in China. Many questions about the etiology and pathogenesis of BPD still remain in spite of a mountain of extensive research that has been done which aimed at identifying risk factors of BPD and planning preventative therapies (5). The foremost predictor for BPD development is prematurity, yet many other factors may contribute.

In order to study the risk factors which could influence the severity of BPD in premature infants and provide reliable bases for effective prevention and control measures, this research conducted a retrospective analysis concerning the clinical data and inspection results interrelated information of 47 cases. In addition, we also hope to develop better practices in the management of newborns with BPD in the future.

\section{Methods}

\subsection{Objects}

These cases were selected from the rare diseases database which was established through the pilot project launched by China (6). There were 51 BPD infants registered in the database in total from 1st January 
2012 to 31 st November 2014. Term infants, cases with congenital anomalies, metabolic or neuromuscular diseases were excluded. Finally, this study consisted of 47 premature infants who were diagnosed with BPD and registered in the database from 2012 to 2014 . There were 33 baby boys (70\%) and 14 baby girls (30\%). All cases corresponded with the BPD diagnosis standard which was formulated by American National Child Health and Human Development Institute (NICHD) in 2000 (7). A total of 28 (59\%) infants had mild BPD, $13(28 \%)$ moderate BPD, and $6(13 \%)$ with severe BPD. The classification standard of severity was also formulated by NICHD (7). Database records included antenatal, natal, and postnatal features together with the laboratory findings of the infants.

\subsection{Data processing and analysis}

SPSS 17.0 was used to input and manage data. Repeated cases have been excluded. Enumeration data was analyzed by chi-square test (Fischer's exact probability test) and measurement data was analyzed by variance analysis. The $95 \%$ confidence interval (CI) and $p$ value were acquired by single factor analysis. In this study, we also used the classification of BPD as dependent variable and all factors with significant associations emerging from the univariate analysis as independent variables to conduct an ordinal logistic regression analysis. The difference was statistically significant when $p<0.05$. Charts were then drawn with SPSS and Excel.

\section{Results}

\subsection{Antenatal features}

As shown in Table 1, the differences were statistically significant when the pregnant mothers had chorioamnionitis and history of abnormal pregnancy. Repeated abortion history, placenta previa, placental abruption, polyhydramnios, oligohydramnios, edemahypertension-proteinuria syndrome, etc were included in the abnormal pregnancy group.

\subsection{Natal features}

Statistical analysis results showed that the smaller the gestational age and the lower the birth weight are, the higher the severity. The results displayed that multiple births didn't have statistical significance despite three sets of twins that suffered from BPD. In the meantime, gender, delivery mode and intrauterine infection pneumonia didn't influence the severity of BPD according to the value of $p$. All the natal features are shown in Table 2.

\subsection{Postnatal characteristics}

Abnormal coagulation function, cholestasis, NRDS and acidosis could affect the severity of BPD among postnatal factors while severity of anemia, HIE, disorders of glucose metabolism and hypoproteinemia were not

Table 1. Conditions of mothers during pregnancies

\begin{tabular}{|c|c|c|c|c|}
\hline Factors & Mild BPD $n(\%)$ & Moderate $\mathrm{BPD} n(\%)$ & Severe BPD $n(\%)$ & $p$ \\
\hline \multicolumn{5}{|l|}{ Maternal age } \\
\hline$\leq 30$ years old & $19(68)$ & $10(77)$ & $5(83)$ & 0.720 \\
\hline $31 \sim 42$ years old & $9(32)$ & $3(23)$ & $1(17)$ & 0.720 \\
\hline Premature rupture of membranes & $10(36)$ & $4(31)$ & $2(33)$ & 1.000 \\
\hline Chorioamnionitis & $7(25)$ & $8(62)$ & $4(67)$ & 0.025 \\
\hline Prenatal hormone application & $4(14)$ & $4(31)$ & $2(33)$ & 0.265 \\
\hline Pregnancy complications & $15(54)$ & $7(54)$ & $5(83)$ & 0.446 \\
\hline History of abnormal pregnancy & $6(21)$ & $6(46)$ & $5(83)$ & 0.009 \\
\hline
\end{tabular}

Table 2. Conditions after the births of infants

\begin{tabular}{|c|c|c|c|c|}
\hline Factors & Mild BPD $n(\%)$ & Moderate $\mathrm{BPD} n(\%)$ & Severe BPD $n(\%)$ & $p$ \\
\hline \multicolumn{5}{|l|}{ Gestational age } \\
\hline$<28$ weeks & $2(7)$ & $1(8)$ & $5(83)$ & \\
\hline $28 \sim 31$ weeks & $21(75)$ & $10(77)$ & $1(17)$ & 0.002 \\
\hline $32 \sim 35+3$ weeks & $5(18)$ & $2(15)$ & 0 & \\
\hline \multicolumn{5}{|l|}{ Birth weight } \\
\hline$<1000 \mathrm{~g}$ & 0 & $4(31)$ & $1(17)$ & \\
\hline $1000 \sim 1499 \mathrm{~g}$ & $21(75)$ & $6(46)$ & $3(50)$ & 0.025 \\
\hline $1500 \sim 1999 \mathrm{~g}$ & $4(14)$ & $3(23)$ & $2(33)$ & \\
\hline $2000 \sim 2015 \mathrm{~g}$ & $3(11)$ & 0 & 0 & \\
\hline Multiple births & $8(29)$ & $2(15)$ & $2(33)$ & 0.626 \\
\hline Baby girl & $7(25)$ & $3(23)$ & $4(67)$ & 0.139 \\
\hline \multicolumn{5}{|l|}{ Delivery mode } \\
\hline Eutocia & $14(50)$ & $8(62)$ & $4(67)$ & 0.701 \\
\hline Cesarean section & $14(50)$ & $5(38)$ & $2(33)$ & \\
\hline Intrauterine infection pneumonia & $8(29)$ & $1(8)$ & 0 & 0.213 \\
\hline
\end{tabular}


Table 3. Clinical manifestations concerning infants in the duration of hospital stay

\begin{tabular}{|c|c|c|c|c|}
\hline Clinical manifestation & Mild BPD $n(\%)$ & Moderate $\mathrm{BPD} n(\%)$ & Severe BPD $n(\%)$ & $p$ \\
\hline \multicolumn{5}{|l|}{ Anemia } \\
\hline Mild & $5(18)$ & $5(39)$ & 0 & \\
\hline Moderate & $17(61)$ & $6(46)$ & $4(67)$ & 0.407 \\
\hline Severe & $6(21)$ & $2(15)$ & $2(33)$ & \\
\hline HIE & $23(82)$ & $10(77)$ & $6(100)$ & 0.555 \\
\hline Disorders of glucose metabolism & $15(54)$ & $6(46)$ & $4(67)$ & 0.704 \\
\hline Hypoproteinemia & $13(46)$ & $3(23)$ & $2(33)$ & 0.394 \\
\hline Abnormal coagulation function & $1(4)$ & $4(31)$ & $4(67)$ & 0.001 \\
\hline Cholestasis & $3(11)$ & $4(31)$ & $4(67)$ & 0.012 \\
\hline PDA & $10(77)$ & $2(22)$ & $2(40)$ & 0.040 \\
\hline Patent foramen ovale & $8(62)$ & $5(56)$ & $3(60)$ & 1.000 \\
\hline NRDS & $12(43)$ & $4(31)$ & $6(100)$ & 0.012 \\
\hline Acidosis & $17(61)$ & $12(92)$ & $6(100)$ & 0.041 \\
\hline
\end{tabular}

HIE, hypoxic-ischemic encephalopathy; PDA, patent ductus arteriosus; NRDS, neonatal respiratory distress syndrome.

Table 4. Ordered logistic regression analysis concerning influence factors of BPD

\begin{tabular}{|c|c|c|c|c|c|c|}
\hline Clinical manifestation & $b^{*}$ & Standard error & Wald & $p$ & OR & $95 \% \mathrm{CI}$ \\
\hline Chorioamnionitis & 3.224 & 0.983 & 10.761 & 0.001 & 25.128 & $1.298-5.150$ \\
\hline Abnormal pregnancy & 1.825 & 0.912 & 4.005 & 0.045 & 6.203 & $0.038-3.613$ \\
\hline Abnormal coagulation function & 2.697 & 1.078 & 6.262 & 0.012 & 14.835 & $0.585-4.809$ \\
\hline Cholestasis & 2.481 & 1.002 & 6.136 & 0.013 & 11.953 & $0.518-4.445$ \\
\hline Gestational age & 3.441 & 1.410 & 5.953 & 0.015 & 31.218 & $0.677-6.204$ \\
\hline Birth weight & 12.523 & 2.293 & 29.856 & 0.0004 & 274580.567 & $8.031-17.051$ \\
\hline
\end{tabular}

${ }^{*}$ Regression coefficient.

influence factors with statistical significance (Table 3). There were 13 mild, 9 moderate and 5 severe cases taken electrocardiographic examinations in total. In the echocardiography results, patent ductus arteriosus could influence severity compared with patent foramen ovale after statistical verification.

\subsection{Ordered logistic regression analysis about influence factors of $B P D$}

Through single factor analysis, 9 factors had statistical significance including: chorioamnionitis, history of abnormal pregnancy, gestational age, birth weight, abnormal coagulation function, cholestasis, NRDS, acidosis and patent ductus arteriosus. In this study, only 27 patients accepted vascular ultrasonography and valid data was too small. Because it didn't meet the conditions of ordered logistic regression analysis, the factor was excluded. By means of ordered logistic regression analysis, chorioamnionitis, history of abnormal pregnancy, abnormal coagulation function, and cholestasis were independent factors which could influence the severity of BPD (Table 4).

\section{Discussion}

After nearly 5 decades since the first description of BPD by Northway, its epidemiology, clinical presentation and pathogenesis have changed. BPD was a chronic lung disease associated with premature birth and still lacked effective prevention and treatment (9). The etiology and pathogenesis were still unclear and most scholars believed that the occurrence and development of BPD were associated with premature birth, inhalation of high concentrations of oxygen, long duration of mechanical ventilation and infection (10). Immature lung development at an early gestational age and light weight infants were the most basic factors for the occurrence of BPD (11-15). This study also showed BPD was more serious when the gestational age was earlier and birth weight was lower owing to immature lung development and respiratory function.

Our study showed that there is a relationship between the severity of BPD and PDA. The incidence of heart failure, pulmonary edema, etc. increased in BPD infants with PDA. It was due to PDA could also significantly increase blood circulation in lung tissue, probability of edema and infection of lung tissue. PDA and BPD may be a cause and effect relationship. Some researchers came to the same conclusion (10). Respiratory distress syndrome (RDS) is a common cause of morbidity and mortality related to premature birth and most infants who develop BPD initially suffer from acute RDS (17). Infants with RDS may easily have acidosis due to adverse pulmonary ventilation. The present study shows, as we expected, that RDS and acidosis are associated with the severity of BPD.

Chorioamnionitis was an independent risk factor in our study. When pregnant women have chorioamnionitis, the expression levels of the proinflammatory cytokines 
increased significantly which could also cause a fetal pulmonary inflammatory reaction or even systemic inflammatory response syndrome. Pulmonary edema, abnormal deposition of fibrin and a decrease of the biological activity of pulmonary surfactant were caused by the pathological process. Inflammatory cytokines in amniotic fluid get into the fetal lung when the mother has chorioamnionitis and caused lung inflammation and injury. The injury continued development after birth and the infants finally developed BPD (18).

Our findings indicated that women with a history of abnormal pregnancy (abortion, stillbirth and premature birth) was another risk factor which could influence the severity of BPD. Some scholars believed that pregnant women with an abnormal childbearing history was a potential risk factor for BPD (19), but yet no studies showed that abnormal pregnancy was an independent risk factor for the severity of BPD. The endometrium cannot fully repair itself in the short term and a cervical mucus embolism that was erased during the operation could lead bacteria easily into the uterine cavity. A common complication is uterine cervical lesions after abortion and pregnant women are prone to premature birth when they have a subsequent pregnancy because the cervix function is not complete. On the other hand, the endometrial myometrial interface was damaged during the artificial abortion and the placental circulation was disordered which could cause placental insufficiency. Finally, these reasons lead to fetal intrauterine hypoxia, slow growth, premature birth and even stillbirth.

Blood vessels are more vulnerable and coagulation factors are deficient in premature infants. Most premature infants had asphyxia when they were born and the function of the organ and adaptability were weaker compared with term infants which could cause pulmonary, intracranial hemorrhage, etc. Therefore, asphyxia could easily cause disorder of blood coagulation function. The development of reticular endothelial structure is imperfect in prematurities. The structure could release tissue factors when the tissue was damaged which was able to initiate the coagulation process. This could further reduce coagulation factors. The situation is more likely to happen when newborns have asphyxia. This is a vicious cycle because hypoxia could accelerate the progress of shock. Therefore, premature infants with abnormal coagulation function and were more commonly applied with mechanical ventilation could further promote the occurrence of BPD. The analytic results in our study also showed abnormal coagulation function was one of the risk factors which could influence the severity of BPD.

We found that cholestasis could influence the severity of BPD, yet no other research has made the same point. Cholestasis means bile acid, bilirubin, etc accumulate in the body. A number of studies supported that severe toxicity reactions occurred in the lungs when the concentration of cholic acid increased $(20,21)$.
Sepulveda found that cholic acid in high concentrations could constrict blood vessels (22) which would lead to a decrease in pulmonary blood flow and even pulmonary hypoxia. Some researchers figured that cholic acid may cause lung damage through hindering the synthesis of pulmonary surfactant and cause an inflammatory reaction $(20,23)$. These above researches explained how cholestasis could injure the development of the lung which may even cause the occurrence of BPD.

\section{Conclusion}

Due to an improvement in the survival of ELBW infants, BPD has been increasing over the past several decades. Because BPD arises from multiple pathogenic processes in the preterm lung which probably cause various results, various aspects of care need meticulous assessment. It originates from the interaction of multiple factors that could injure the immature lung, and for exactly that reason, preventions must be developed on the basis of all the factors implicated in its pathogenesis. The more serious the illness is, the worse the prognosis. Severe BPD could even influence the brain or other vital organs in the long term process. Therefore, we can actively take preventive measures to control disease development according to potential risk factors in the clinical performance.

Our data showed that the most relevant risk factors that could influence the severity of BPD were low birth weight, early gestational age, chorioamnionitis, childbearing history, cholestasis and abnormal coagulation function. Comprehending the case history, especially whether the mother had chorioamnionitis or an abnormal pregnancy history, and careful examination were vital. Mothers who have had abortions many times may give birth to severe BPD infants to a greater extent. The birth of premature infants with low birth weight and early gestational age are more likely to develop severe BPD (24). Paying close observation to the appearance of cholestasis and abnormal coagulation function could control and prevent the progress of the severity of BPD. It is needed that all NICUs keep making efforts to know better practices, decrease risk factors and contribute to the prevention of BPD.

\section{Acknowledgements}

This study was supported by The Key Projects in the National Science \& Technology Pillar Program during the Twelfth Five-year Plan Period (2013BAI07B01).

\section{References}

1. Northway WH Jr, Rosan RC, Porter DY. Pulmonary disease following respirator therapy of hyaline-membrane disease: Bronchopulmonary dysplasia. N Engl J Med. 1967; 276:357-368. 
2. Walsh MC, Yao Q, Gettner P, et al. Impact of a physiologic definition on bronchopulmonary dysplasia rates. Pediatrics. 2004; 114:1305-1311.

3. Abman SH, Groothius JR. Pathophysiology and treatment of bronchopulmonary dysplasia. Current issues. Pediatr Clin North Am. 1994; 41:277-315.

4. Monte LF, Silva Filho LV, Miyoshi MH, Rozov T. Bronchopulmonary dysplasia. J Pediatr. 2005; 81:99-110.

5. Eduardo Bancalari. What is the evidence for drug therapy in the prevention and management of bronchopulmonary dysplasia? The newborn lung: Neonatal questions and controversies. 1st ed. Saunders Elsevier, Amsterdam, Netherlands, 2008; pp. 208-232.

6. Cui Y, Zhou X, Han J. China launched a pilot project to improve its rare disease healthcare levels. Orphanet J Rare Dis. 2014; 9:14.

7. Jobe AH, Bancalari E. Bronchopulmonary dysplasia. Am J Respir Crit Care Med. 2001; 163:1723-1729.

8. Wang $\mathrm{H}, \mathrm{Mu} \mathrm{DZ}$. The treatment and clinical application about drug of bronchopulmonary dysplasia. Chin J Obstet Gynecol Pediatr. 2010; 6:290-295. (in Chinese)

9. Groothuis JR, Makari D. Definition and outpatient management of the very low-birth-weight infant with bronchopulmonary dysplasia. Adv Ther. 2012; 29:297311.

10. Zhang H,Fu JH, Xue XD. The analysis on high risk factors about bronchopulmonary dysplasia in premature infants with ventilation. Chinese Pediatric Emergency Medicine. 2011; 3:240-242. (in Chinese)

11. Capoluongo E, Ameglio F, Lulli P, Minucci A, Santonocito C, Concolino P, Di Stasio E, Boccacci S, Vendettuoli V, Giuratrabocchetta G, De Cunto A, Tana M, Romagnoli C, Zuppi C, Vento G. Epithelial lining fluid free IGF-I-to-PAPP-A ratio is associated with bronchopulmonary dysplasia in preterm infants. Am J Physiol Endocrinol Metab. 2007; 292:e308-e313.

12. Grisaru-Granovsky S, Reichman B, Lerner-Geva L, Hammerman C, Samueloff A, Schimmel MS; Israel Neonatal Network. Mortality and morbidity in preterm small-for-gestational-age infants: A population-based study. Am J Obstet Gynecol. 2012; 206:150.e1-e7.

13. Bhering CA, Mochdece CC, Moreira ME, Rocco JR, Sant'Anna GM. Bronchopulmonary dysplasia prediction model for 7-day-old infants. J Pediatr. 2007; 83:163-170.
14. Baraldi E, Filippone M. Chronic lung disease after premature birth. N Engl J Med. 2007; 357:1946-1955.

15. Kinsella JP, Greenough A, Abman SH. Bronchopulmonary dysplasia. Lancet. 2006; 367:1421-1431.

16. Stephens BE, Gargus RA, Walden RV, Mance M, Nye J, McKinley L, Tucker R, Vohr BR. Fluid regimens in the first week of life may increase risk of patent ductus arteriosus in extremely low birthweight infants. J Perinatol. 2008; 28:123-128.

17. Gursoy T, Hayran M, Derin H, Ovali F. A clinical scoring system to predict the development of bronchopulmonary dysplasia. Am J Perinatol. 2014. (DOI: 10.1055/s-00341393935)

18. Watterberg KL, Demers LM, Scott SM, Murphy S. Chorioamnionitis and early lung inflammation in infants in whom bronchopulmonary dysplasia develops. Pediatrics. 1996; 97:210-215.

19. Walsh MC, Szefler S, Davis J, Allen M, Van Marter L, Abman S, Blackmon L, Jobe A. Summary proceedings from the bronchopulmonary dysplasia group. Pediatrics. 2006; 117:s52-s56.

20. Zecca E, De Luca D, Baroni S, Vento G, Tiberi E, Romagnoli C. Bile acid-induced lung injury in newborn infants: A bronchoalveolar lavage fluid study. Pediatrics. 2008; 121:e146-e149.

21. Why does intrahepatic cholestasis of pregnancy could cause lung injury in newborn? (http://www.jkb.com.cn) xunyiwenyao/other/2013/0130/21414.html) (accessed December 20, 2014). (in Chinese)

22. Sepúlveda WH, González C, Cruz MA, Rudolph MI. Vasoconstrictive effect of bile acids on isolated human placental chorionic veins. Eur J Obstet Gynecol Reprod Biol. 1991; 42:211-215.

23. Hills BA, Chen Y, Masters IB, Hills YC. Raised bile acid concentrations in SIDS lungs at necropsy. Arch Dis Child. 1997; 77:120-123.

24. Eriksson L, Haglund B, Odlind V, Altman M, Ewald U, Kieler H. Perinatal conditions related to growth restriction and inflammation are associated with an increased risk of bronchopulmonary dysplasia. Acta Paediatr. 2015; 104:259-263.

(Received January 13, 2015; Revised March 10, 2015; Accepted March 16, 2015) 